Rodríguez-Ferran, A., Morata, I. and Huerta, A., A new damage model based on nonlocal displacements, International Journal for Numerical and Analytical

Methods in Geomechanics, Vol. 29, Issue 5, pp. 473-493, 2005

\title{
A new damage model based on nonlocal displacements
}

\author{
Antonio Rodríguez-Ferran*, Irene Morata and Antonio Huerta \\ Laboratori de Càlcul Numèric (LaCàN) \\ Edifici C2, Campus Nord, Universitat Politècnica de Catalunya \\ E-08034 Barcelona, Spain. \\ e-mail:\{antonio.rodriguez-ferran,irene.morata,antonio.huerta\}@upc.es \\ web page: www-lacan.upc.es
}

KEY WORDS: nonlocal damage models; nonlocal displacements; gradient models; consistent tangent matrix; quadratic convergence

\section{SUMMARY}

A new nonlocal damage model is presented. Nonlocality (of integral or gradient type) is incorporated into the model by means of nonlocal displacements. This contrasts with existing damage models, where a nonlocal strain or strain-related state variable is used. The new model is very attractive from a computational viewpoint, especially regarding the computation of the consistent tangent matrix needed to achieve quadratic convergence in Newton iterations. At the same time, its physical response is very similar to that of the standard models, including its regularization capabilities. All these aspects are discussed in detail and illustrated by means of numerical examples.

\section{INTRODUCTION}

Nonlocal damage models are used to model failure of quasi-brittle materials [1]. Nonlocality -needed to correct the pathological mesh-dependence exhibited by local models- can be incorporated into the model in two different ways. In integral-type models $[2,3,4]$, a nonlocal state variable is computed as the weighted average of the local state variable in a neighbourhood of the point under consideration. In gradient-type models [5], on the other hand, higher-order derivatives (typically second-order) are added to the partial differential equation that describes the evolution of the nonlocal variable. Both approaches yield similar results and are in some cases equivalent [6].

Apart from the state variable, other variables can be selected to incorporate nonlocality. Either scalar or tensorial quantities may be transformed into the corresponding nonlocal

*Correspondence to: Antonio Rodríguez-Ferran, Departament de Matemàtica Aplicada III, E.T.S. d'Enginyers de Camins. Edifici C2, Campus Nord, Universitat Politècnica de Catalunya. E-08034 Barcelona, Spain.

Contract/grant sponsor: Ministerio de Educación y Ciencia; contract/grant number: DPI2004-03000 
quantities. In fact, a number of proposals can be found in the literature. For integral-type regularization, some examples are the use of a nonlocal damage parameter [3], nonlocal strains [7] or nonlocal strain invariants [8]. These and other existing approaches are compared in [9] by means of a simple 1D numerical test (bar under uniaxial tension). Various approaches are also possible for gradient regularization. In [10], for instance, the loading function depends on the Laplacian of the damage parameter. Note that all these strategies involve Gauss-point based quantities.

A new proposal is made here: to use nonlocal displacements to regularize the problem. The two versions are proposed, discussed and compared: integral-type (nonlocal displacements obtained as the weighted average of standard, local displacements), see [11], and gradienttype (nonlocal displacements obtained as the solution of a second-order PDE). As discussed and illustrated by means of numerical examples, the regularization capabilities of this new model are very similar to that of the standard model. In addition, it is very attractive from a computational viewpoint, especially regarding (1) the computation of the consistent tangent matrix and (2) the simple and straightforward upgrade of a nonlinear FE code to account for nonlocality.

An outline of this paper follows. The basic features of standard nonlocal damage models are reviewed in Section 2. The new model based on nonlocal displacements is presented in Section 3. The integral-type and gradient versions are discussed in Sections 3.1 and 3.2 respectively. The regularization capabilities are illustrated by means of a uniaxial tension test. Section 4 deals with the consistent linearization of the nonlinear equilibrium equation. It is shown how
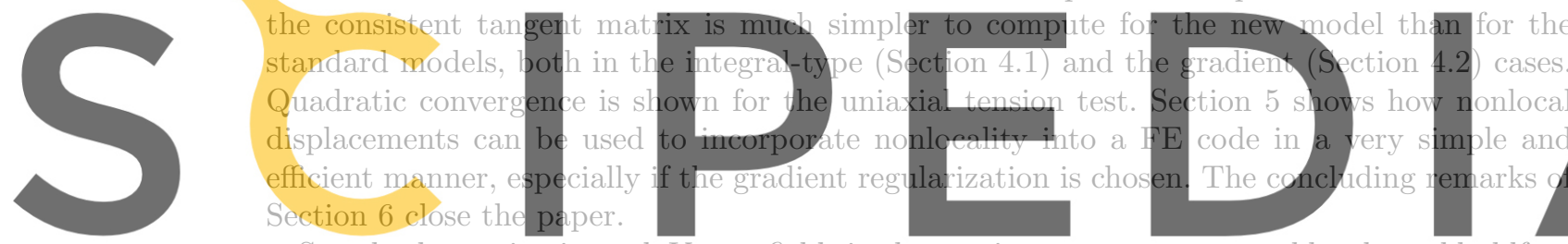

Standard notation is used. Vector fields in the continuum are represented by slanted boldface type (u: displacement field.). Nodal vectors associated to FE discretization are denoted by

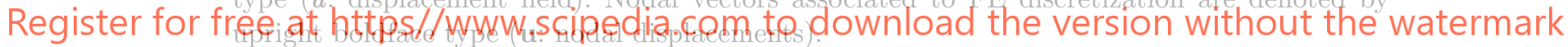

\section{OVERVIEW OF DAMAGE MODELS}

For simplicity, only elastic-scalar damage models are considered here. However, the concept of nonlocal displacements can be extended to more complex damage models exhibiting, for instance, anisotropy or plasticity $[4,12]$.

\subsection{Local damage models}

A generic local damage model consists of the following equations, summarized in table I:

- A relation between Cauchy stresses $\sigma$ and small strains $\varepsilon$-i.e. the symmetrized gradient of displacements $u$, Equation (2) - , where the loss of stiffness (from elastic stiffness $\mathbf{C}$ to zero stiffness) is described by means of a scalar damage parameter $D$ which ranges from 0 to 1, Equation (1);

- The definition of a local state variable $Y$ as a function of strain $\varepsilon$, Equation (3); 
- A damage evolution law, where the local state variable $Y$ drives the evolution of the non-decreasing damage parameter $D$, Equation (4).

Table I. General expression of a local damage model

$$
\begin{aligned}
\text { Stress-strain relationship } & \sigma(x, t)=(1-D(x, t)) \mathbf{C} \varepsilon(x, t) \\
\text { Strains } & \varepsilon(\boldsymbol{x}, t)=\nabla^{s} u(\boldsymbol{x}, t) \\
\text { Local state variable } & Y(x, t)=Y(\varepsilon(x, t)) \\
\text { Damage evolution } & D(\boldsymbol{x}, t)=D\left(\max _{\tau \leq t} Y(\boldsymbol{x}, \tau)\right)
\end{aligned}
$$

The most common particular forms of Equations (3) and (4) are reviewed in [11]. Regarding the definition of the state variable, both the so-called Mazars model and modified von Mises model result in $Y=\varepsilon$ in the simple case of uniaxial tension. As for damage evolution, the linear softening law is especially suited for conceptual analyses. Between the damage threshold $Y_{0}$ (inception of damage) and a maximum admissible value $Y_{\mathrm{f}}(D=1)$, damage evolves according
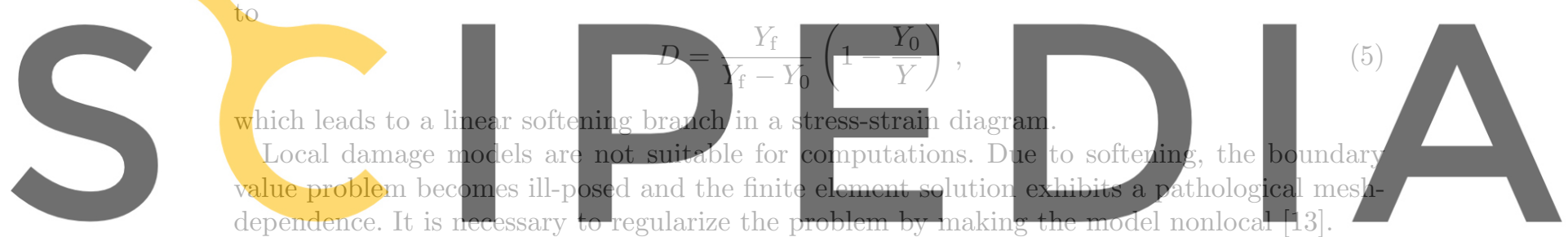

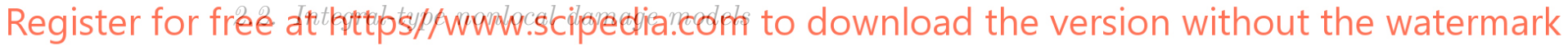

In integral-type models, see table II, nonlocality is incorporated via the definition of a nonlocal state variable $\tilde{Y}$ as the weighted average of the local state variable $Y$, Equation (6). The weighting function $\alpha$ depends on the distance $r$ between two points and contains a characteristic length $l_{\mathrm{c}}$ as a parameter, Equation (7). The nonlocal state variable $\tilde{Y}$, rather than the local state variable $Y$, drives the evolution of damage, Equation (8).

The weighting function $\alpha$ is typically defined as

$$
\alpha\left(\boldsymbol{x}, r ; l_{\mathrm{c}}\right)=c_{0}(\boldsymbol{x}) \alpha_{\star}\left(r ; l_{\mathrm{c}}\right),
$$

where $\alpha_{\star}$ is the Gaussian function $[14,15,16]$

$$
\alpha_{\star}\left(r ; l_{\mathrm{c}}\right)=\exp \left[-\left(\frac{2 r}{l_{\mathrm{c}}}\right)^{2}\right]
$$

and the normalization factor $c_{0}(\boldsymbol{x})$ is

$$
c_{0}(x)=1 / \int_{V_{x}} \alpha_{\star}\left(r ; l_{\mathrm{c}}\right) \mathrm{d} z
$$


Table II. General expression of an integral-type nonlocal damage model

Stress-strain relationship

Strains

Local state variable

Nonlocal state variable

Weighting function

Damage evolution

$$
\begin{aligned}
& \sigma(x, t)=(1-D(x, t)) \mathbf{C} \varepsilon(x, t) \\
& \varepsilon(\boldsymbol{x}, t)=\nabla^{s} \boldsymbol{u}(\boldsymbol{x}, t)
\end{aligned}
$$$$
Y(x, t)=Y(\varepsilon(x, t))
$$$$
\tilde{Y}(x, t)=\int_{V_{x}} \alpha(x, z) Y(z, t) \mathrm{d} z
$$

$$
\begin{aligned}
& \alpha(x, z)=\alpha\left(x, r ; l_{\mathrm{c}}\right) \text { with } r=\|x-z\| \\
& D(x, t)=D\left(\max _{\tau<t} \tilde{Y}(x, \tau)\right)
\end{aligned}
$$
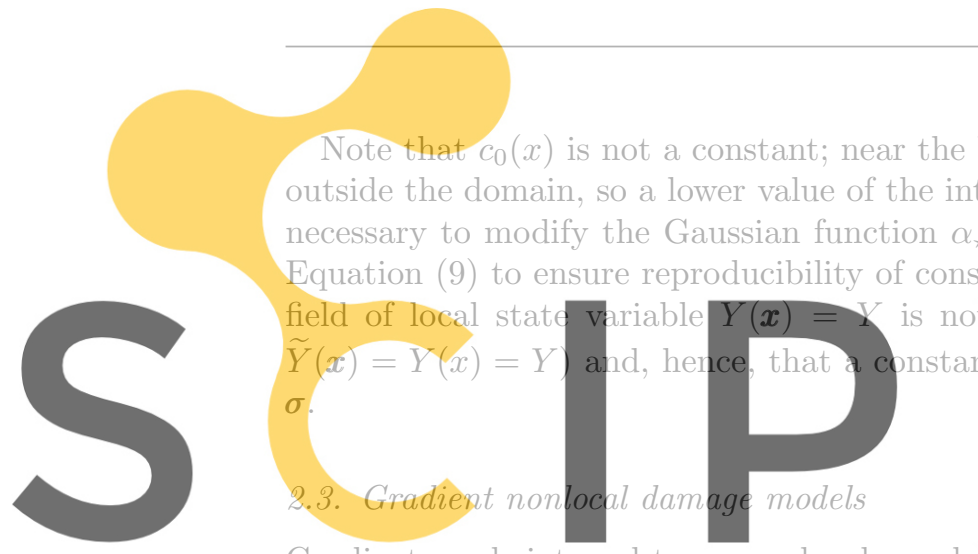

Note that $c_{0}(x)$ is not a constant; near the boundaries, the support of $\alpha_{\star}$ may lay partially outside the domain, so a lower value of the integral in Equation (11) is obtained. In fact, it is necessary to modify the Gaussian function $\alpha_{\star}$ into the weighting function $\alpha$ as indicated by Equation (9) to ensure reproducibility of constant functions. This guarantees that a constant field of local stat
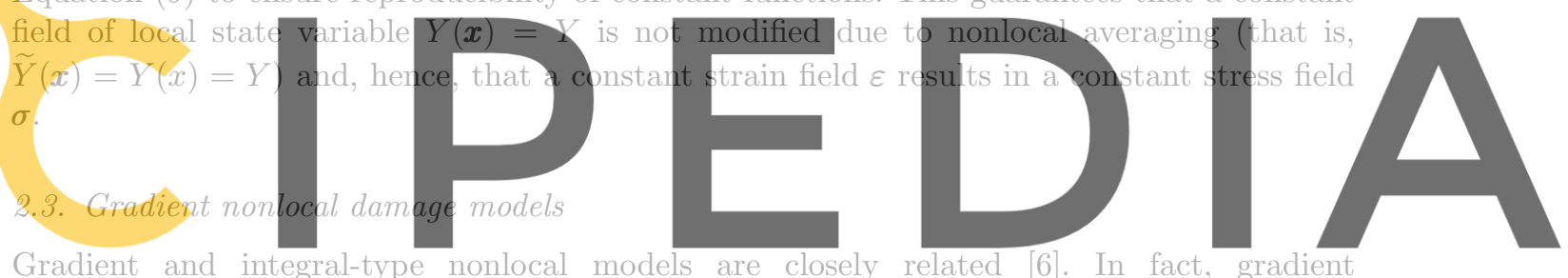

regularization can be regarded as an approximation to integral-type regularization, with a

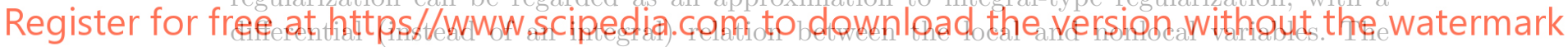

gradient enhancement may be explicit or implicit [17]. In implicit gradient models [5] the nonlocal state variable $\widetilde{Y}$ is the solution of a partial differential equation with the local state variable $Y$ as the source term. Various studies $[18,17]$ show that the second-order implicit gradient enhancement provides the best approximation to the integral-type nonlocal model. For this reason, we have chosen this particular gradient approach to facilitate our comparative analysis. The regularization PDE is in this case a diffusion-reaction equation, see Equation (12) in table III. Note that a characteristic length is incorporated into the model via parameter $c$ in the diffusion term.

The definition of appropriate boundary conditions for the regularization equation (12) is a debated issue in the development of gradient models. Homogeneous Neumann boundary conditions are typically prescribed, see Equation (13). Application of the divergence theorem shows that this condition implies that the total amount of the state variable does not change due to regularization, $\int_{\Omega} \widetilde{Y} \mathrm{~d} \Omega=\int_{\Omega} Y \mathrm{~d} \Omega$, see [18]. However, the main reasons for choosing this type of condition were mathematical convenience and the difficulties to motivate Dirichlet boundary conditions (i.e. prescribing $\tilde{Y}$ ). Only very recently the homogeneous Neumann boundary conditions have been interpreted, from a thermodynamical viewpoint, 
as an insulation condition in the so-called nonlocality energy residual [19].

Table III. General expression of a gradient nonlocal damage model

Stress-strain relationship

Strains

Local state variable

Nonlocal state variable

Damage evolution

$$
\boldsymbol{\sigma}(\boldsymbol{x}, t)=(1-D(\boldsymbol{x}, t)) \mathbf{C} \varepsilon(\boldsymbol{x}, t)
$$$$
\varepsilon(x, t)=\nabla^{s} \boldsymbol{u}(x, t)
$$$$
Y(\boldsymbol{x}, t)=Y(\varepsilon(\boldsymbol{x}, t))
$$$$
\tilde{Y}(\boldsymbol{x}, t)-c \nabla^{2} \widetilde{Y}(\boldsymbol{x}, t)=Y(x, t) \text { in } \Omega
$$$$
\mathbf{n} \nabla \tilde{Y}=0 \text { on } \partial \Omega
$$

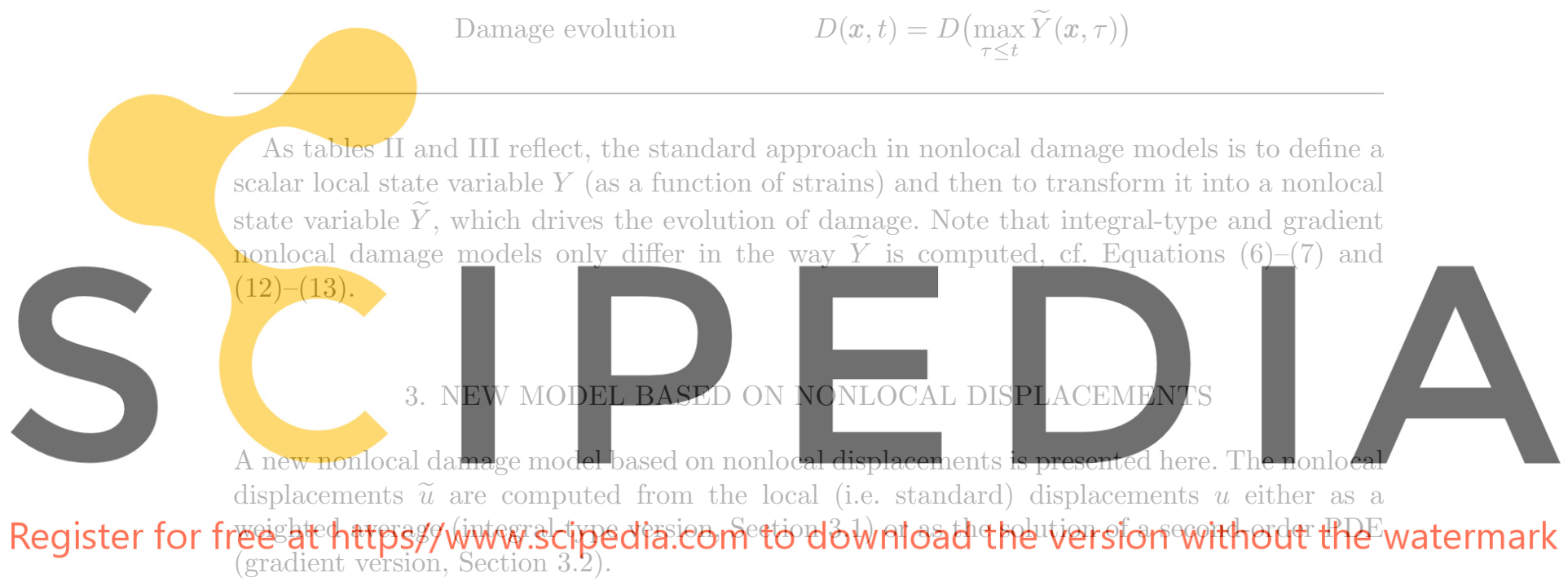

\subsection{Integral-type version}

The integral-type version of the proposed model is summarized in table IV. The key idea is the computation of nonlocal displacements as the weighted average of local displacements, Equation (14). After that, the nonlocal strains $\varepsilon_{\mathrm{NL}}$, the nonlocal state variable $Y_{\mathrm{NL}}$ and, finally, the damage parameter $D$ are obtained. Note that these three variables are computed locally: nonlocality is introduced at the "beginning" of the constitutive model (i.e. at the level of displacements, the primal unknowns in the FE computation).

Regarding the basic ingredients of a nonlocal damage model reviewed in Section 2, the only one that requires some modification is the weighting function. Since displacements, rather than strains, are averaged, reproducibility of polynomials of degree 1 is needed to ensure that a constant strain field results in a constant stress field. This can be done in a simple and computationally efficient manner, as described next.

Moving least-squares fitting, a standard technique in meshless methods [20, 21], suggests how to define the weighting function. To ensure reproducibility of order 0 , the kernel $\alpha_{\star}$ is 
Table IV. New model based on nonlocal displacements, integral-type version. Subscript NL denotes quantities with nonlocal information but computed locally.

Stress-strain relationship

Local strains

Nonlocal displacements

Nonlocal strains

Nonlocal state variable

Damage evolution

$$
\begin{aligned}
& \sigma(x, t)=(1-D(x, t)) \mathbf{C} \varepsilon(x, t) \\
& \varepsilon(\boldsymbol{x}, t)=\nabla^{s} \boldsymbol{u}(\boldsymbol{x}, t) \\
& \widetilde{u}(\boldsymbol{x}, t)=\int_{V_{x}} \widehat{\alpha}(\boldsymbol{x}, z) u(\boldsymbol{z}, t) \mathrm{d} z \\
& \varepsilon_{\mathrm{NL}}(\boldsymbol{x}, t)=\nabla^{s} \widetilde{\boldsymbol{u}}(\boldsymbol{x}, t) \\
& Y_{\mathrm{NL}}(x, t)=Y\left(\varepsilon_{\mathrm{NL}}(x, t)\right) \\
& D(x, t)=D\left(\max _{\tau \leq t} Y_{\mathrm{NL}}(x, \tau)\right)
\end{aligned}
$$

multiplied by a polynomial of order 0 in the neighbourhood of each point $x$, see Equation (9).

For reproducibility of order 1 , more degrees of freedom are needed, so a polynomial of order 1 is needed,

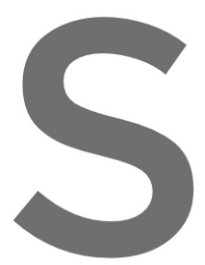

$$
\widehat{\alpha}(x, z)=\left[\widehat{c}_{0}(x)+z^{T} \widehat{c}_{1}(x)\right] \alpha_{\star}(x-z) .
$$
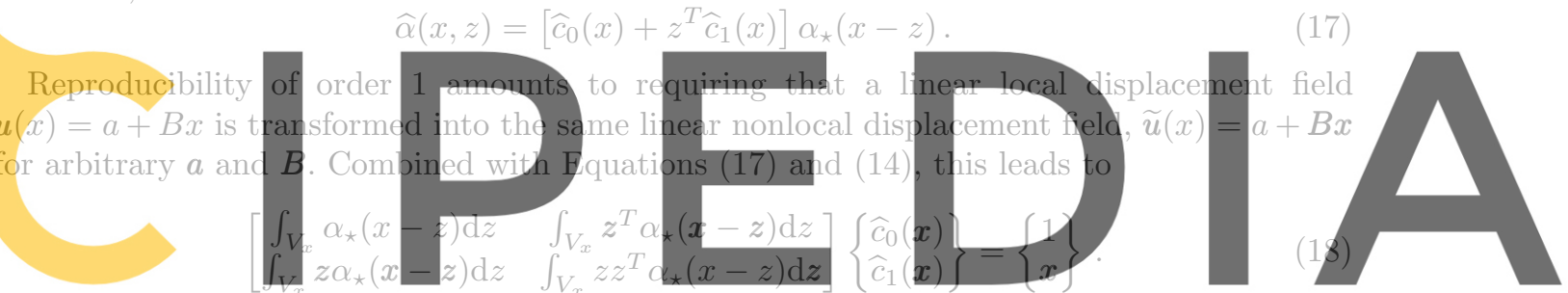

This small system of linear equations (order: number of space dimensions +1 ) needs to be

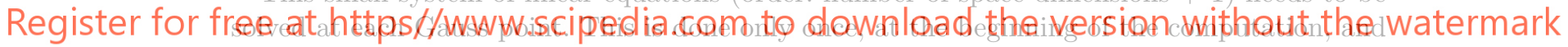
coefficients $\widehat{c}_{0}(x)$ and $\widehat{c}_{1}(x)$ are stored and reused throughout the analysis.

Is the weighting function $\widehat{\alpha}$ different from the weighting function of the standard approach,

Equation (9)? Yes, but only near the boundaries.

Away from the boundaries (that is, when volume $V_{x}$ is not truncated by the domain boundary), the off-diagonal terms in the matrix of Equation (18) may be expressed as

$$
\int_{V_{x}} z \alpha_{\star}(x-z) \mathrm{d} z=\underbrace{\int_{V_{x}}(z-x) \alpha_{\star}(x-z) \mathrm{d} z}_{I=0}+\boldsymbol{x} \int_{V_{x}} \alpha_{\star}(\boldsymbol{x}-\boldsymbol{z}) \mathrm{d} z .
$$

The first integral in the RHS of Equation (19) is null due to the symmetry of function $\alpha_{\star}$ and integration domain $V_{x}$ and the skew-symmetry of function $z-x$. Equation (19) implies that, in the linear system (18), the first matrix column is proportional to the RHS vector, so $\widehat{\boldsymbol{c}}_{1}(\boldsymbol{x})=\mathbf{0}, \widehat{c}_{0}(\boldsymbol{x})=c_{0}(x)$ and $\widehat{\alpha}(x, z)=\alpha(x, z)$.

Near the boundary, on the other hand, the first integral in the RHS of Equation (19) is not zero, because the truncated integration domain $V_{x}$ is not symmetric, so $\widehat{c}_{1}(x) \neq \mathbf{0}$, $\widehat{c}_{0}(x) \neq c_{0}(x)$ and $\widehat{\alpha}(x, z) \neq \alpha(x, z)$. 
In other words: for the new model based on nonlocal displacements, it is only necessary to modify the weighting function in the Gauss points near the boundaries. Away from the boundaries, reproducibility of order 0 automatically implies reproducibility of order 1 .

\subsection{Gradient version}

The gradient version of the proposed model is summarized in table $\mathrm{V}$. The second-order PDE now relates nonlocal displacements $\widetilde{u}$ to local displacements $u$, see Equation (20). Note that Dirichlet boundary conditions (21) are prescribed for $\widetilde{\boldsymbol{u}}$. These boundary conditions have a clear physical interpretation: nonlocal displacements must coincide with local displacements in all the domain boundary (i.e. for both the Dirichlet and Neumann boundaries of the mechanical problem). Another important difference between the gradient enhancements of the state variable and the displacements are the continuity and interpolation requirements. Upon finite element discretization, the local state variable $Y$ in Equation (12) is a discontinuous (piecewise polynomial, Gauss-point-based) field, but $\mathcal{C}^{0}$ continuity is required for the (nodalbased) nonlocal state variable field $\widetilde{Y}$. In Equation (20), on the contrary, both $u$ and $\widetilde{u}$ are $\mathcal{C}^{0}$ nodal based fields. In fact, the choice of interpolation functions for the two fields $(\boldsymbol{u}$ and $\widetilde{Y}$ ) in a standard gradient-enhanced damage model is a debated issue, see [22]; for the new model, on the other hand, it is very natural to use the same interpolation functions for the two displacement fields $u$ and $\widetilde{u}$.
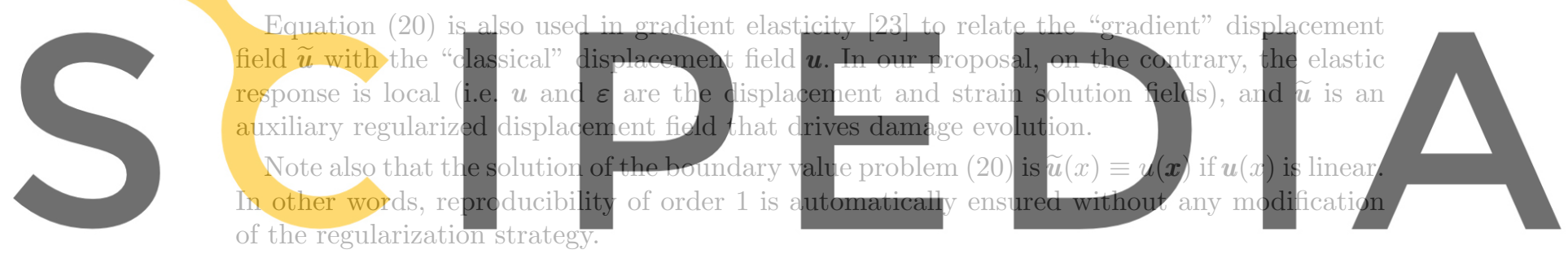

\section{Register for free at https//www.scipedia.com to download the version without the watermark}

Table V. New model based on nonlocal displacements, gradient version. Subscript NL denotes quantities with nonlocal information but computed locally.

Stress-strain relationship

Local strains

Nonlocal displacements

Nonlocal strains

Nonlocal state variable

Damage evolution

$$
\begin{aligned}
& \sigma(x, t)=(1-D(x, t)) \mathbf{C} \varepsilon(x, t) \\
& \varepsilon(x, t)=\nabla^{s} u(x, t) \\
& \widetilde{u}(x, t)-c \nabla^{2} \widetilde{u}(x, t)=u(x, t) \text { in } \Omega \\
& \widetilde{u}=u \text { on } \partial \Omega \\
& \varepsilon_{\mathrm{NL}}(x, t)=\nabla^{s} \widetilde{\boldsymbol{u}}(x, t) \\
& Y_{\mathrm{NL}}(x, t)=Y\left(\varepsilon_{\mathrm{NL}}(\boldsymbol{x}, t)\right) \\
& D(\boldsymbol{x}, t)=D\left(\max _{\tau \leq t} Y_{\mathrm{NL}}(x, \tau)\right)
\end{aligned}
$$




\subsection{Validation of the new model}

The regularization capabilities of the model based on nonlocal displacements are illustrated here by means of a uniaxial tension test, see Figure 1(a) and [22].

The one-dimensional particularizations of the integral-type and gradient versions, tables IV and $\mathrm{V}$, with $Y(\varepsilon) \equiv \varepsilon$ and a linear softening law, see Figure 1(b), are used. As a reference, the standard integral-type model with nonlocal state variable (i.e. nonlocal strains, since $Y \equiv \varepsilon$ ) is taken.

The central tenth of the bar is weakened (10\% reduction in Young's modulus) to trigger localization. The dimensionless geometric and material parameters are summarized in table VI. The numerical tests are displacement-controlled.
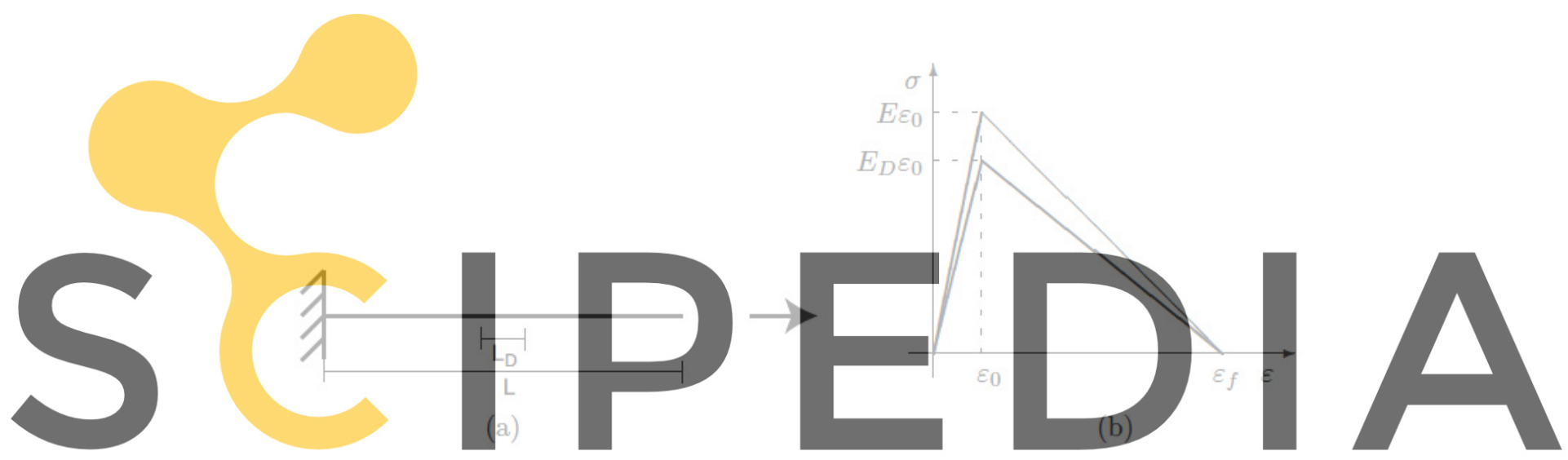

Figure 1. Uniaxial tension test: (a) problem statement; (b) linear softening law Register for free at https//www.scipedia.com to download the version without the watermark

\begin{tabular}{|c|c|c|}
\hline Meaning & Symbol & Value \\
\hline Length of bar & $L$ & 100 \\
\hline Cross-section of bar & $A$ & 1 \\
\hline Idem of weaker part & $L_{D}$ & 10 \\
\hline Young's modulus & $E$ & 20000 \\
\hline Idem of weaker part & $E_{D}$ & 18000 \\
\hline Damage threshold & $\varepsilon_{0}$ & $10^{-4}$ \\
\hline Final strain & $\varepsilon_{f}$ & $1.25 \times 10^{-2}$ \\
\hline
\end{tabular}

The regularization properties of the model based on nonlocal displacements are assessed first by carrying out the analysis with five different meshes of $40,80,160,320$ and 640 elements (corresponding respectively to element sizes $h$ of $2.5,1.25,0.625,0.3125$ and 0.15625 ).

For the integral-type version, a fixed characteristic length $l_{\mathrm{c}}=6.25$ (corresponding respectively to $2.5,5,10,20$ and 40 elements) is chosen. The results for the various meshes 
are shown in Figure 2. Both the force-displacement curves and the final damage profiles are virtually superimposed.
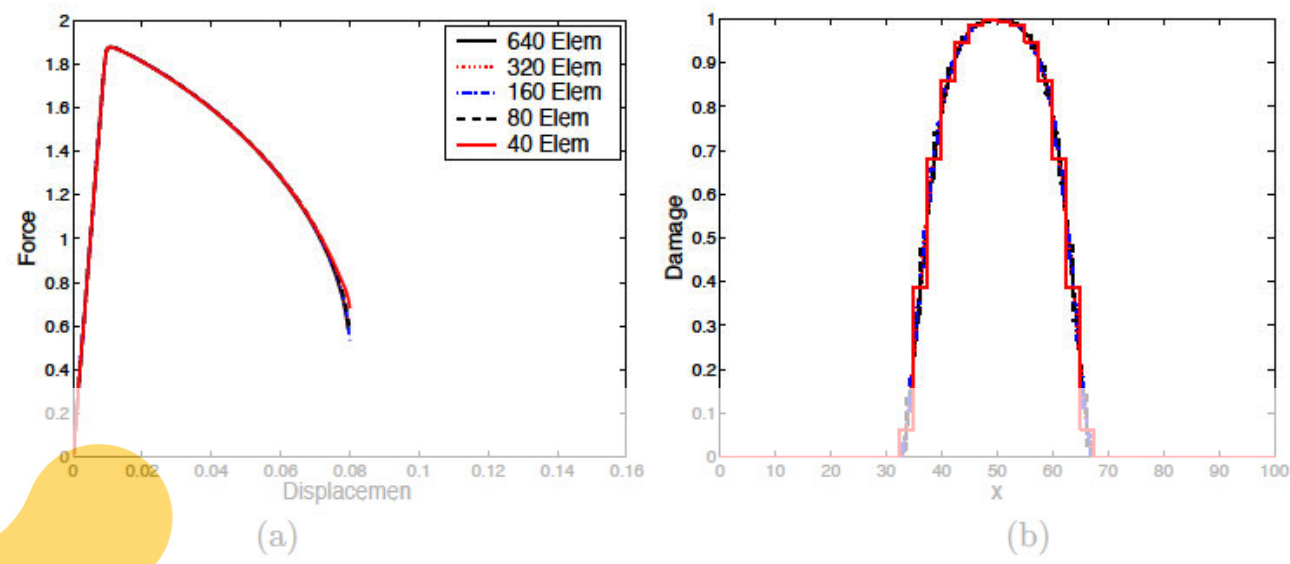

(b)

Figure 2. New model based on nonlocal displacements, integral-type version: (a) force-displacement and (b) final damage profiles for various meshes

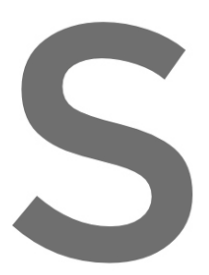

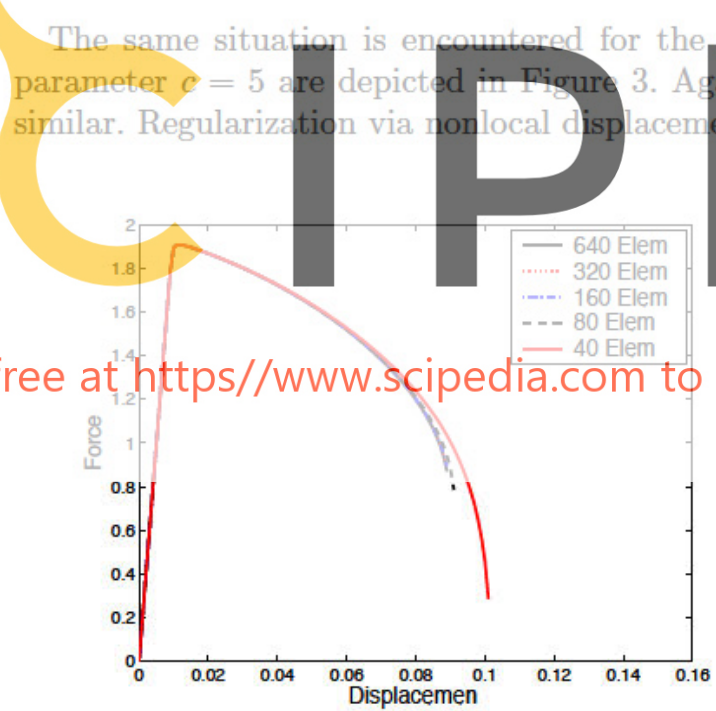

(a)

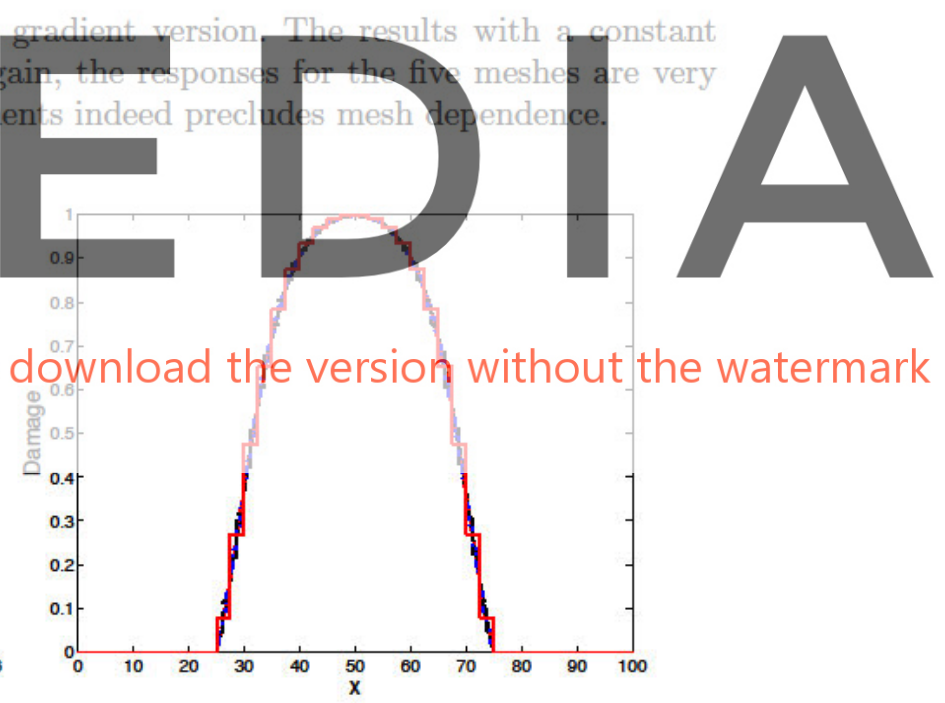

(b)

Figure 3. New model based on nonlocal displacements, gradient version: (a) force-displacement and (b) final damage profiles for various meshes

As a second test, a fixed mesh of 320 elements $(h=0.3125)$ and four different internal lengths are used. For the integral-type model, characteristic lengths $l_{\mathrm{c}}$ of $5 h, 10 h, 20 h=6.25$ (reference value above) and $40 h$ are taken; for the gradient model, parameters $c$ of $1,2,5$ (reference) and 10. 
The results are depicted in Figures 4 and 5. As desired, both the ductility in the forcedisplacement response and the width of the final damage profile increase with the internal length scale.
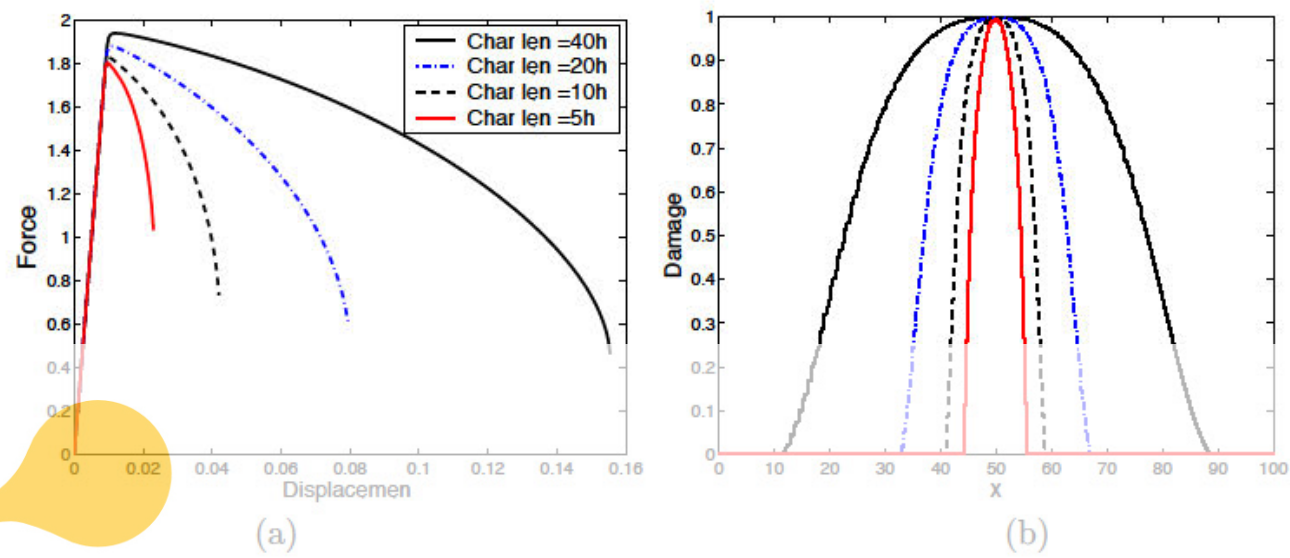

(b)

Figure 4. New model based on nonlocal displacements, integral-type version: (a) force-displacement and (b) final damage profiles for various characteristic lengths

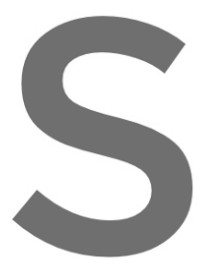

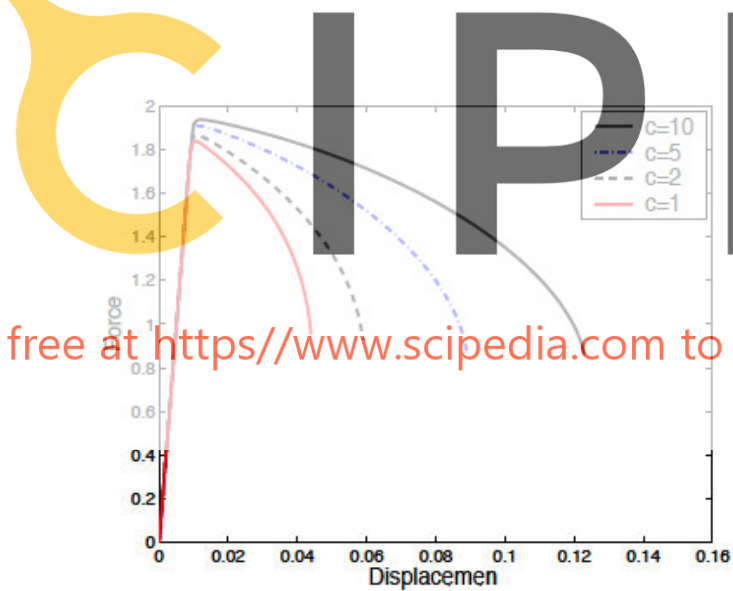

(a)
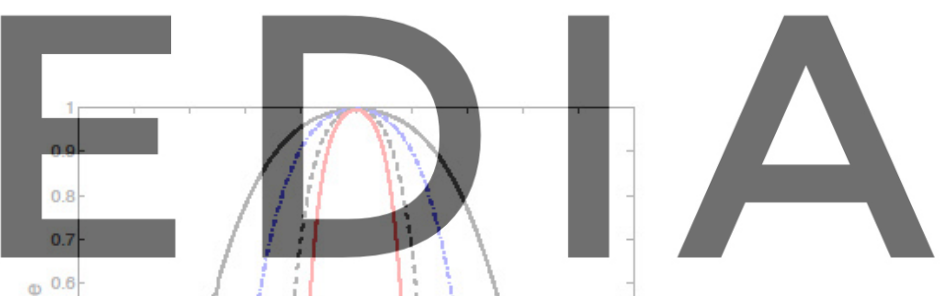

Register for free at https//www.scipedia.com to do.jownload the versián without the watermark

Figure 5. New model based on nonlocal displacements, gradient version: (a) force-displacement and

(b) final damage profiles for various parameters $c$

To sum up: the new model exhibits the same regularization capabilities and qualitative response than the standard model based on nonlocal strains.

A quantitative comparison is carried out next, for the integral-type version. The test with 320 elements and $l_{\mathrm{c}}=6.25$ is analyzed in detail. Apart from the same structural response, see Figure 6(a), the two models also predict the same evolution of damage profile, see Figure 6(b), and the same stress-strain diagram for three sample points, see Figure 6(c). 


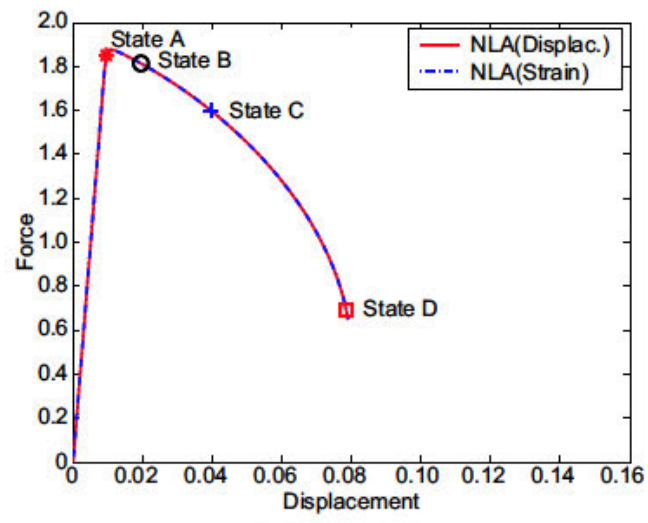

(a)
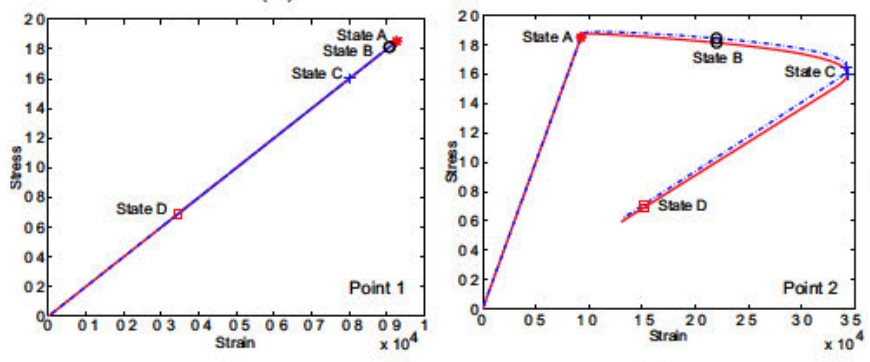

(c)

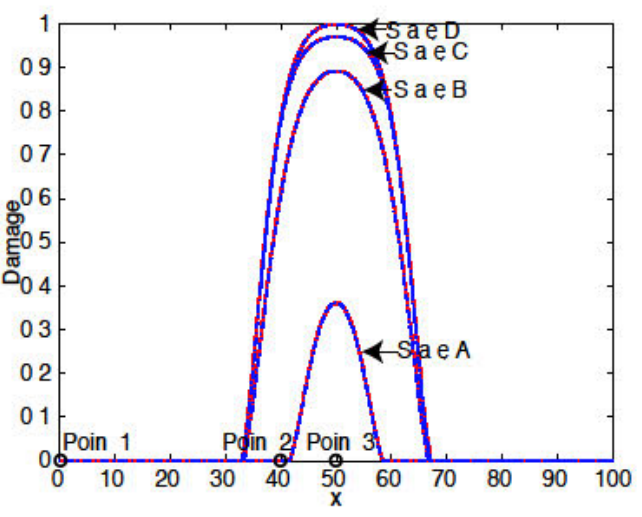

(b)

Figure 6. Integral-type versions. The new model (nonlocal displacements) and the standard model (nonlocal strains) yield very similar results: (a) force-displacement curve; (b) damage profiles; (c) stress-strain diagrams at sample points.

\subsection{Connection between the standard and the new model}

In this section the similarities between the two models are outlined. The goal is to demonstrate that the proposed approach based on nonlocal displacements inherits the regularization properties of the standard model. The connection between the two approaches is especially clear for the one-dimensional particularization used above (where the state variable is simply the strain, $Y \equiv \varepsilon$ ).

For the integral-type version, this connection relies on the fact that convolution (i.e. weighted average) and differentiation commute in an infinite domain. For the gradient version, the two models are equivalent. Differentiation of the regularization equation in terms of displacements, Equation (20), leads directly to the usual equation in terms of strains, Equation (12). Moreover, the Dirichlet boundary conditions on displacements and the homogeneous Neumann boundary conditions on strains are equivalent: substitution of Equation (21) into Equation (20) results in $\mathrm{d}^{2} \widetilde{u} / \mathrm{d} x^{2}=\mathrm{d} \varepsilon_{\mathrm{NL}} / \mathrm{d} x=0$.

The effect of boundary conditions over finite domains (for integral-type regularization) and a multidimensional setting - where the state variable $Y$ is a nonlinear scalar function of the strain tensor $\varepsilon-$ (for both integral-type and gradient approaches) will be analyzed in detail in a forthcoming contribution. 


\section{CONSISTENT LINEARIZATION}

The new model based on nonlocal displacements is very attractive from a computational viewpoint, especially regarding its consistent linearization (i.e. the computation of the consistent tangent matrix needed to attain quadratic convergence in the full Newton-Raphson method [24]). For both regularization approaches, we first review the expression of the consistent tangent matrix of the standard model (nonlocal state variable) reported in the literature. After that, we present the counterpart for the new model based on nonlocal displacements and show that it is much simpler to compute.

\subsection{Consistent tangent matrix for integral-type models}

Integral-type models pose a substantial difficulty: due to nonlocality, there is interaction between non-adjacent nodes, and the consistent tangent matrix exhibits a larger bandwidth (with respect to the sparsity pattern of the elastic or secant matrices) [25, 26, 11].

In FE analysis, the internal force vector is typically computed with a Gauss quadrature as

$$
\mathbf{f}_{\mathrm{int}}(\mathbf{u})=\sum_{p} w_{p} \mathbf{B}_{p}^{T} \sigma_{p}(\mathbf{u})
$$

where $p$ ranges the Gauss points, $w_{p}$ are the corresponding integration weights, $\mathbf{B}_{p}$ is the usual matrix of shape function derivatives at Gauss point $p$ and stresses $\sigma_{p}$ are

$$
\boldsymbol{\sigma}_{p}(\mathbf{u})=\left(1-D_{p}(\mathbf{u})\right) \mathbf{C} \underbrace{\mathbf{B}_{p} \mathbf{u}}_{\varepsilon_{p}}
$$

The consistent tangent matrix is

$$
\mathbf{K}_{\mathrm{tan}}:=\frac{\partial \mathbf{f}_{\mathrm{int}}}{\partial \mathbf{u}}=\sum_{p} w_{p} \mathbf{B}_{p}^{T} \frac{\partial \boldsymbol{\sigma}_{p}}{\partial \mathbf{u}} .
$$

Combining Equations (23) and (24) results in

$$
\mathbf{K}_{\mathrm{tan}}=\mathbf{K}_{\mathrm{sec}}+\mathbf{K}_{\text {nonlocal }}
$$

where

$$
\mathbf{K}_{\mathrm{sec}}=\sum_{p} w_{p} \mathbf{B}_{p}^{T}\left(1-D_{p}\right) \mathbf{C B}_{p}
$$

is the secant stiffness matrix and

$$
\mathbf{K}_{\text {nonlocal }}=-\sum_{p} w_{p} \mathbf{B}_{p}^{T} \mathbf{C} \boldsymbol{\varepsilon}_{p} \frac{\partial D_{p}}{\partial \mathbf{u}}
$$

is the nonlocal tangent contribution which accounts for the variation of the damage parameter.

Equations (22)-(27) are general. The specific structure of matrix $\mathbf{K}_{\text {nonlocal }}$, however, is quite different in the standard and new models. 
4.1.1. Standard model (table II) By applying the chain rule, the term $\partial D_{p} / \partial \mathbf{u}$ in Equation (27) can be expressed as

$$
\frac{\partial D_{p}}{\partial \mathbf{u}}=D^{\prime}\left(\widetilde{Y}_{p}\right) \frac{\partial \widetilde{Y}_{p}}{\partial \mathbf{u}}
$$

The integral (6) in table II required for nonlocal averaging is also approximated via a numerical quadrature, so the nonlocal state variable $\widetilde{Y}_{p}$ is

$$
\widetilde{Y}_{p}=\sum_{q \in V_{p}} w_{q} \alpha_{p q} Y_{q}
$$

where $q$ ranges the Gauss points $\xi_{q}$ in the neighbourhood $V_{p}$ of Gauss point $\xi_{p}$, and $\alpha_{p q}=\alpha\left(r=\left\|\xi_{p}-\xi_{q}\right\|\right)$.

By differentiating Equation (29), the last term in Equation (28) can be expressed as

$$
\frac{\partial \tilde{Y}_{p}}{\partial \mathbf{u}}=\sum_{q \in V_{p}} w_{q} \alpha_{p q} \frac{\partial Y_{q}}{\partial \mathbf{u}}=\sum_{q \in V_{p}} w_{q} \alpha_{p q} \frac{\partial Y_{q}}{\partial \varepsilon} \mathbf{B}_{q}
$$

where the chain rule and the relation $\partial \varepsilon_{q} / \partial \mathbf{u}=\mathbf{B}_{q}$ have been used.

By replacing Equation (30) into Equation (28) and then into Equation (27), the nonlocal matrix can be expressed as

$$
\mathbf{K}_{\text {nonlocal }, Y}=-\sum_{p, q \in V_{p}} w_{p q} \mathbf{B}_{p}^{T} \mathbf{C} \varepsilon_{p} D^{\prime}\left(\tilde{Y}_{p}\right) \frac{\partial Y_{q}}{\partial \varepsilon} \mathbf{B}_{q}
$$

where $w_{p q}=w_{p} w_{q} \alpha_{p q}$ and the subscript $Y$ denotes the nonlocal quantity. Due to the double loop in Gauss points caused by nonlocal interaction, $\mathbf{K}_{\text {nonlocal, } Y}$ cannot be assembled from elementary contributions solely.

4.1.2. New model (table IV) Equations (22)-(27) are also valid for the new model. However, the term $\partial D_{p} / \partial \mathbf{u}$ is now

$$
\frac{\partial D_{p}}{\partial \mathbf{u}}=D^{\prime}\left(Y_{\mathrm{NL}_{p}}\right) \frac{\partial Y}{\partial \boldsymbol{\varepsilon}_{\mathrm{NL}}}\left(\varepsilon_{\mathrm{NL}_{p}}\right) \frac{\partial \varepsilon_{\mathrm{NL}_{p}}}{\partial \widetilde{\mathbf{u}}} \frac{\partial \widetilde{\mathbf{u}}}{\partial \mathbf{u}}
$$

Since nonlocal averaging is performed at the beginning, the rest of the constitutive model is "local". Note, in particular, that the usual shape functions are used in the FE discretization of nonlocal displacements and that nonlocal strains $\varepsilon_{\mathrm{NL}}$ are computed locally as the symmetrized gradient of nonlocal displacements, see table IV. This means that

$$
\varepsilon_{\mathrm{NL}_{p}}=\mathbf{B}_{p} \widetilde{\mathbf{u}} \quad \Longrightarrow \quad \frac{\partial \varepsilon_{\mathrm{NL}_{p}}}{\partial \widetilde{\mathbf{u}}}=\mathbf{B}_{p}
$$

where $\mathbf{B}_{p}$ is the same matrix of shape function derivatives used in Equation (22).

The last term in $(32), \partial \widetilde{\mathbf{u}} / \partial \mathbf{u}$, reflects the nonlocality of the model. After finite element discretization and numerical integration, the averaging process (14) leads simply to

$$
\widetilde{\mathbf{u}}=\mathbf{A}_{\text {integral }} \mathbf{u} \quad \Longrightarrow \quad \frac{\partial \widetilde{\mathbf{u}}}{\partial \mathbf{u}}=\mathbf{A}_{\text {integral }},
$$


where $\mathbf{A}_{\text {integral }}$ is a matrix of nonlocal connectivity. Note that this matrix contains for standard models where the characteristic length $l_{\mathrm{c}}$ or parameter $c$ are not evolutiondependent - purely geometrical information associated to the finite element mesh. It does not change as damage evolves, so it can be computed and stored at the beginning of the analysis (provided, of course, that a fixed mesh is used).

Substitution of Equation (32), (33) and (34) into Equation (27) results in

$$
\mathbf{K}_{\text {nonlocal, } \mathbf{u}}=\mathbf{K}_{\text {local, }} \mathbf{u} \mathbf{A}_{\text {integral }}
$$

with

$$
\mathbf{K}_{\text {local }, \mathbf{u}}=-\sum_{p} w_{p} \mathbf{B}_{p}^{T} \mathbf{C} \boldsymbol{\varepsilon}_{p} D^{\prime}\left(Y_{\mathrm{NL}_{p}}\right) \frac{\partial Y}{\partial \varepsilon_{\mathrm{NL}}}\left(\varepsilon_{\mathrm{NL}_{p}}\right) \mathbf{B}_{p}
$$

Note that $\mathbf{K}_{\text {local, }}$ can be computed in the usual way by assembling elementary matrices, like in any local material model. After that, nonlocality is accounted for by means of the constant matrix $\mathbf{A}_{\text {integral }}$, which "spreads" the stiffness of $\mathbf{K}_{\text {local, } \mathbf{u}}$ into $\mathbf{K}_{\text {nonlocal, }}$.

By replacing Equation (35) in Equation (25), the consistent tangent matrix can be expressed as

$$
\mathbf{K}_{\text {tan }}=\mathbf{K}_{\text {sec }}+\mathbf{K}_{\text {local }} \mathbf{A}_{\text {integral }}
$$

This simple structure of $\mathbf{K}_{\mathrm{tan}}$ is due to the fact that the nonlocal average is performed completely "upstream" in the constitutive equation (i.e. with displacements, the primal unknowns in the FE analysis). For any other choice of the nonlocal variable, see Section 1, double loops in Gauss points like those in Equation (31) appear.

Equation (37) is graphically illustrated by Figure 7, where the pattern of the consistent tangent matrix for the four representative load steps of Figure 6(a). Note how the constant matrix $\mathbf{A}_{\text {integral }}$ indeed "spreads" the local stiffness matrix $\mathbf{K}_{\text {local }}$ (which is non-zero only where damage increases), thus provoking fill-in in the tangent matrix $\mathbf{K}_{\tan }$.

The convergence history at these four load steps is shown in Figure 8. As expected, only linear convergence is achieved with the secant stiffness matrix, while the consistent tangent matrix leads to quadratic convergence.

\subsection{Consistent tangent matrix for gradient models}

Gradient models are mathematically local. Nonlocal interaction is accounted for locally via higher-order spatial derivatives. Thanks to this, the consistent tangent matrix is simpler to compute than for integral nonlocal models.

Gradient models are typically formulated as two-field problems. For the standard model, the unknowns are the nodal vectors of displacements $\mathbf{u}$ and nonlocal state variables $\widetilde{\mathbf{Y}}$. For the new model, on the other hand, unknowns are the nodal vectors of local and nonlocal displacements, $\mathbf{u}$ and $\widetilde{\mathbf{u}}$.

4.2.1. Standard model (table III) Finite element discretization leads to

$$
\begin{aligned}
\mathbf{r}_{\text {equil }}(\mathbf{u}, \tilde{\mathbf{Y}}) & :=\mathbf{f}_{\text {int }}(\mathbf{u}, \tilde{\mathbf{Y}})-\mathbf{f}_{\text {ext }}=\mathbf{0} \\
\mathbf{r}_{\text {regu }}(\mathbf{u}, \tilde{\mathbf{Y}}) & :=(\mathbf{M}+c \mathbf{D}) \tilde{\mathbf{Y}}-\mathbf{f}_{Y}(\mathbf{u})=\mathbf{0}
\end{aligned}
$$



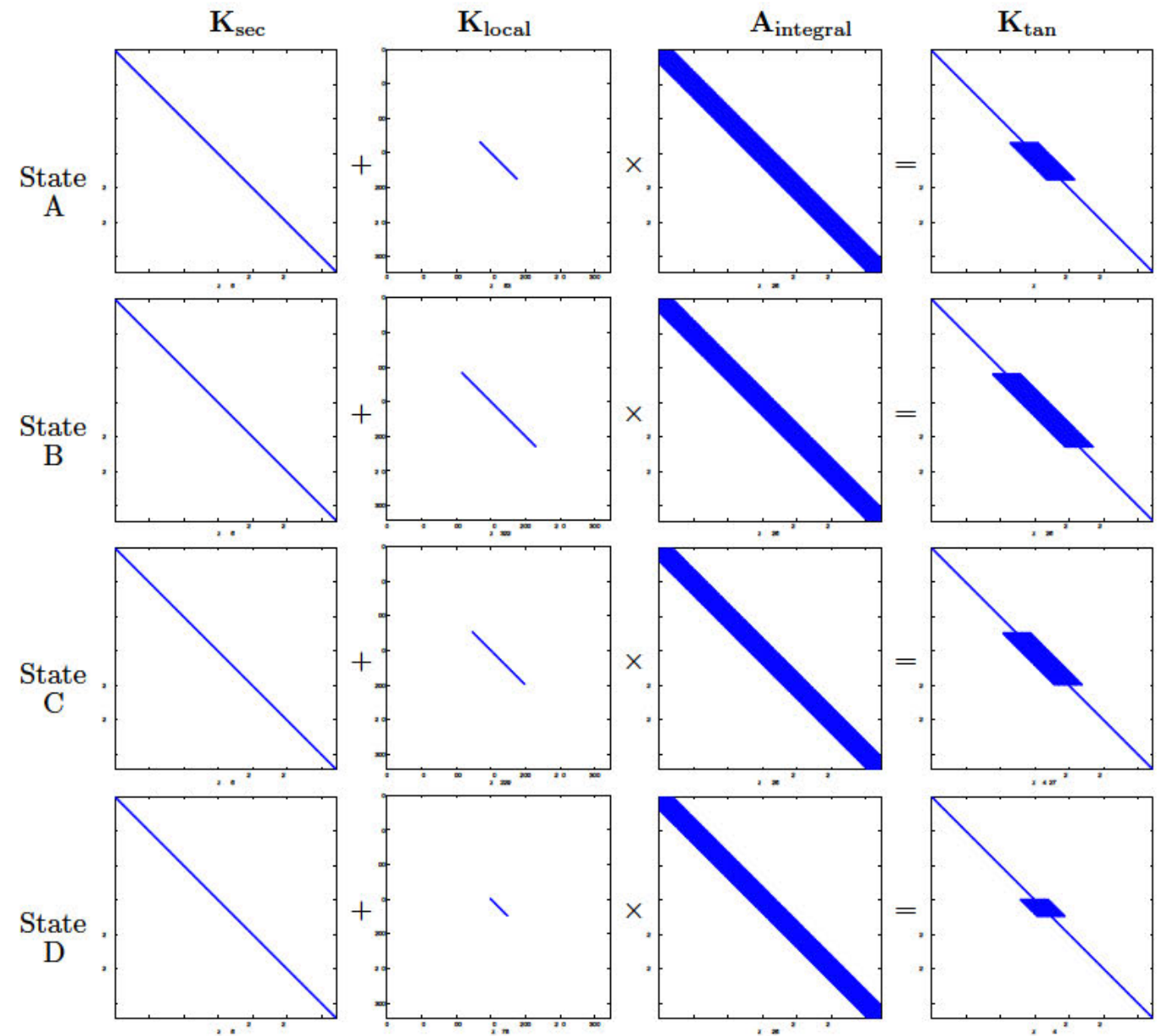

Figure 7. Integral-type model based on nonlocal displacements. Sparsity pattern of the consistent tangent matrix at load steps A, B, C and D, see Figure 6(a)

Equation (38) is the nonlinear system of equilibrium equations. Since $\mathbf{u}$ and $\widetilde{\mathbf{Y}}$ are treated as independent variables, the vector of internal forces is now

$$
\mathbf{f}_{\text {int }}(\mathbf{u}, \tilde{\mathbf{Y}})=\sum_{p} w_{p} \mathbf{B}_{p}^{T} \sigma_{p}(\mathbf{u}, \tilde{\mathbf{Y}})
$$

with stresses

$$
\boldsymbol{\sigma}_{p}(\mathbf{u}, \widetilde{\mathbf{Y}})=\left(1-D_{p}(\widetilde{\mathbf{Y}})\right) \mathbf{C B}_{p} \mathbf{u} .
$$

Equation (39) is the discretized weak form of the diffusion-reaction PDE (12). The mass and diffusivity matrices are

$$
\mathbf{M}=\int_{\Omega} \mathbf{N}^{T} \mathbf{N d} V \quad \text { and } \quad \mathbf{D}=\int_{\Omega} \nabla \mathbf{N}^{T} \nabla \mathbf{N d} V
$$



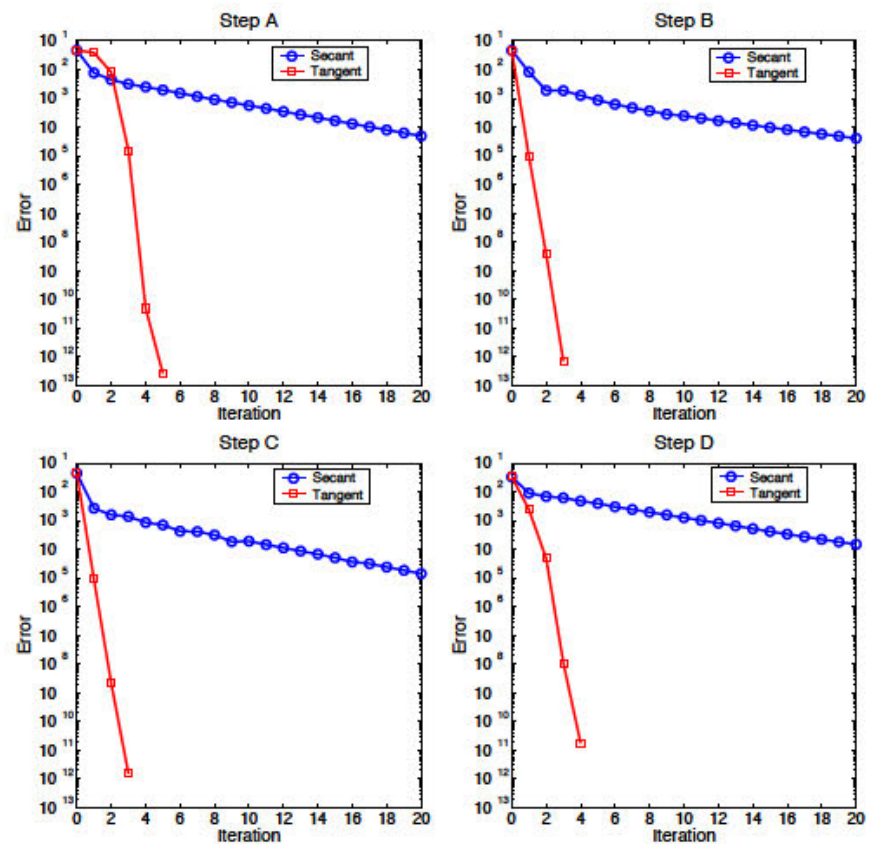

Figure 8. Integral-type model based on nonlocal displacements. Convergence history of the secant matrix and the consistent tangent matrix for load steps A, B, C and D, see Figure 6(a)

and the "force" vector is

$$
\mathbf{f}_{Y}=\sum_{p} w_{p} \mathbf{N}_{p}^{T} Y_{p}(\mathbf{u})
$$

with $\mathbf{N}$ the matrix of shape functions and $\nabla \mathbf{N}$ the matrix of shape function gradients.

Linearization of Equations (38) and (39) results in the tangent matrix

$$
\mathbf{K}_{\tan }=\left[\begin{array}{ll}
\mathbf{K}_{\mathbf{u u}} & \mathbf{K}_{\mathbf{u} \tilde{\mathbf{Y}}} \\
\mathbf{K}_{\tilde{\mathbf{Y}} \mathbf{u}} & \mathbf{K}_{\tilde{\mathbf{Y}} \tilde{\mathbf{Y}}}
\end{array}\right]
$$

with

$$
\begin{aligned}
\mathbf{K}_{\mathbf{u u}} & :=\frac{\partial \mathbf{r}_{\text {equil }}}{\partial \mathbf{u}}=\sum_{p} w_{p} \mathbf{B}_{p}^{T}\left(1-D_{p}\right) \mathbf{C B}_{p} \\
\mathbf{K}_{\mathbf{u} \tilde{\mathbf{Y}}} & :=\frac{\partial \mathbf{r}_{\text {equil }}}{\partial \widetilde{\mathbf{Y}}}=-\sum_{p} w_{p} \mathbf{B}_{p}^{T} \mathbf{C} \varepsilon_{p} D^{\prime}\left(\widetilde{Y}_{p}\right) \mathbf{N}_{p} \\
\mathbf{K}_{\tilde{\mathbf{Y}} \mathbf{u}} & :=\frac{\partial \mathbf{r}_{\text {regu }}}{\partial \mathbf{u}}=-\sum_{p} w_{p} \mathbf{N}_{p}^{T} \frac{\partial Y}{\partial \varepsilon}\left(\varepsilon_{p}\right) \mathbf{B}_{p} \\
\mathbf{K}_{\tilde{\mathbf{Y}} \tilde{\mathbf{Y}}} & :=\frac{\partial \mathbf{r}_{\text {regu }}}{\partial \widetilde{\mathbf{Y}}}=\mathbf{M}+c \mathbf{D},
\end{aligned}
$$

so a Newton iteration is

$$
\left[\begin{array}{ll}
\mathbf{K}_{\mathbf{u u}}^{i} & \mathbf{K}_{\mathbf{u} \tilde{\mathbf{Y}}}^{i} \\
\mathbf{K}_{\tilde{\mathbf{Y}} \mathbf{u}}^{i} & \mathbf{K}_{\tilde{\mathbf{Y}} \tilde{\mathbf{Y}}}
\end{array}\right]\left\{\begin{array}{l}
\delta \mathbf{u}^{i+1} \\
\delta \widetilde{\mathbf{Y}}^{i+1}
\end{array}\right\}=\left\{\begin{array}{l}
-\mathbf{r}_{\text {equil }}^{i} \\
-\mathbf{r}_{\text {regu }}^{i}
\end{array}\right\}
$$


where $i$ is the iteration counter. Note that $\mathbf{K}_{\mathbf{u u}}^{i}$ is the secant stiffness matrix, cf. Equations (45) and (26), and that $\mathbf{K}_{\tilde{\mathbf{Y}} \tilde{\mathbf{Y}}}$ is a constant matrix.

4.2.2. New model (table $V$ ) The two fields are now u and $\widetilde{\mathbf{u}}$. Finite element discretization results in

$$
\begin{aligned}
\mathbf{r}_{\text {equil }}(\mathbf{u}, \widetilde{\mathbf{u}}) & :=\mathbf{f}_{\text {int }}(\mathbf{u}, \widetilde{\mathbf{u}})-\mathbf{f}_{\text {ext }}=\mathbf{0} \\
\mathbf{r}_{\text {regu }}(\mathbf{u}, \widetilde{\mathbf{u}}) & :=-\mathbf{M u}+(\mathbf{M}+c \mathbf{D}) \widetilde{\mathbf{u}}=\mathbf{0}
\end{aligned}
$$

where Equation (51) is a linear system associated to the linear diffusion-reaction equation (20).

The consistent tangent matrix is

$$
\mathbf{K}_{\tan }=\left[\begin{array}{ll}
\mathbf{K}_{\mathbf{u u}} & \mathbf{K}_{\mathbf{u} \widetilde{\mathbf{u}}} \\
\mathbf{K}_{\widetilde{\mathbf{u} u}} & \mathbf{K}_{\widetilde{\mathbf{u}} \mathbf{u}}
\end{array}\right]
$$

with

$$
\begin{aligned}
& \mathbf{K}_{\mathbf{u u}}:=\frac{\partial \mathbf{r}_{\text {equil }}}{\partial \mathbf{u}}=\sum_{p} w_{p} \mathbf{B}_{p}^{T}\left(1-D_{p}\right) \mathbf{C B}_{p} \\
& \mathbf{K}_{\mathbf{u} \widetilde{\mathbf{u}}}:=\frac{\partial \mathbf{r}_{\text {equil }}}{\partial \widetilde{\mathbf{u}}}=-\sum_{p} w_{p} \mathbf{B}_{p}^{T} \mathbf{C} \varepsilon_{p} D^{\prime}\left(Y_{\mathrm{NL}_{p}}\right) \frac{\partial Y}{\partial \varepsilon_{\mathrm{NL}}}\left(\varepsilon_{\mathrm{NL}_{p}}\right) \mathbf{B}_{p} \\
& \mathbf{K}_{\widetilde{\mathbf{u} u}}:=\frac{\partial \mathbf{r}_{\mathrm{regu}}}{\partial \mathbf{u}}=-\mathbf{M} \\
& \mathbf{K}_{\widetilde{\mathbf{u}} \widetilde{\mathbf{u}}}:=\frac{\partial \mathbf{r}_{\mathrm{regu}}}{\partial \widetilde{\mathbf{u}}}=\mathbf{M}+c \mathbf{D},
\end{aligned}
$$

so a Newton iteration is

$$
\left[\begin{array}{ll}
\mathbf{K}_{\mathbf{u u}}^{i} & \mathbf{K}_{\mathbf{u} \tilde{\mathbf{u}}}^{i} \\
\mathbf{K}_{\widetilde{\mathbf{u} u}} & \mathbf{K}_{\widetilde{\mathbf{u}} \mathbf{u}}
\end{array}\right]\left\{\begin{array}{l}
\delta \mathbf{u}^{i+1} \\
\delta \widetilde{\mathbf{u}}^{i+1}
\end{array}\right\}=\left\{\begin{array}{c}
-\mathbf{r}_{\text {equil }}^{i} \\
\mathbf{0}
\end{array}\right\} .
$$

Some remarks about the tangent matrix (52):

- Matrices $\mathbf{K}_{\mathbf{u u}}^{i}$ and $\mathbf{K}_{\mathbf{u} \mathbf{u}}^{i}$ are the secant and the local tangent matrices already obtained for the integral-type version, cf. Equations (53) and (54) with Equations (26) and (36).

- Matrices $\mathbf{K}_{\tilde{\mathbf{u}} \mathbf{u}}$ and $\mathbf{K}_{\tilde{\mathbf{u}} \tilde{\mathbf{u}}}$ are both constant, due to the linearity of the regularization Equation (51). This fact can be effectively exploited if a quasi-Newton solver is preferred over the Newton-Raphson method for equilibrium iterations, see [27].

- Thanks also to the linear relation between $\mathbf{u}$ and $\widetilde{\mathbf{u}}$, the residual $\mathbf{r}_{\text {regu }}$ is zero.

Figure 9 shows the convergence history at four representative steps (similar to those chosen for the integral-type model). Again, quadratic convergence is only achieved with the tangent stiffness matrix.

\subsection{One-field gradient version}

Thanks to the linear relation between local and nonlocal displacements, the gradient version ca be re-formulated as a one-field problem, which highlights the close connection between the gradient and integral regularizations. 

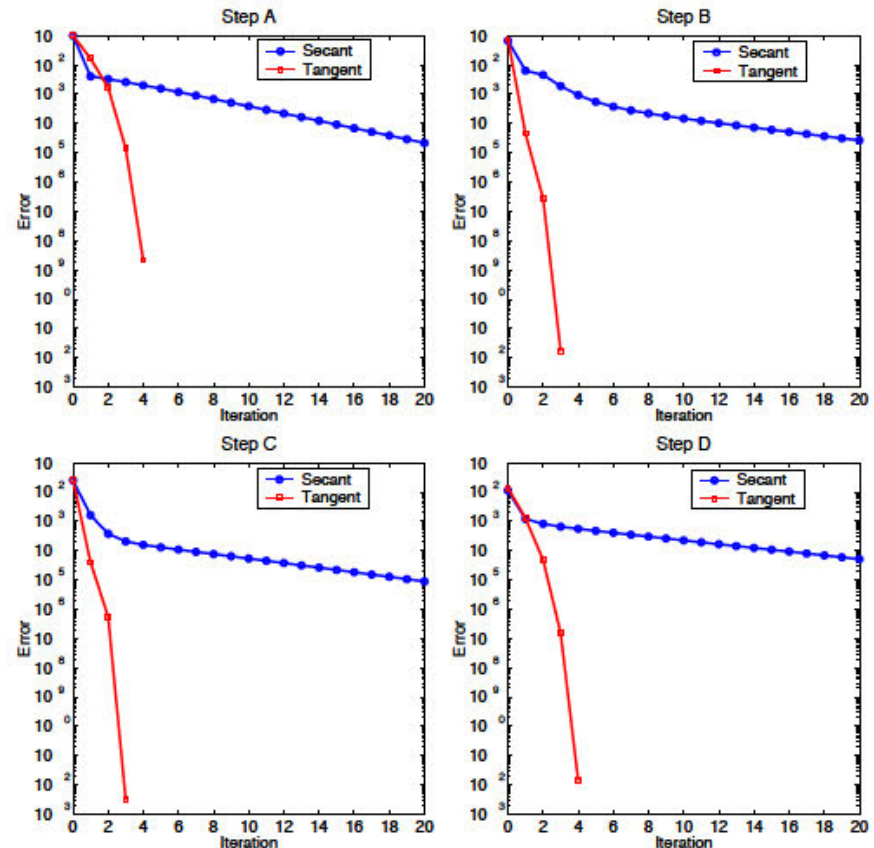

Figure 9. Gradient model based on nonlocal displacements. Convergence history of the secant matrix and the consistent tangent matrix for four representative load steps

Equation (51) can be inverted into

$$
\widetilde{\mathbf{u}}=\mathbf{A}_{\text {gradient }} \mathbf{u} \quad \text { with } \quad \mathbf{A}_{\text {gradient }}=(\mathbf{M}+c \mathbf{D})^{-1} \mathbf{M}=\left(\mathbf{I}+c \mathbf{M}^{-1} \mathbf{D}\right)^{-1}
$$

where $\mathbf{A}_{\text {gradient }}$ is, like $\mathbf{A}_{\text {integral }}$ for the integral nonlocal version, a constant matrix with purely geometrical information.

Matrix $\mathbf{A}_{\text {gradient }}$ clearly reflects the implicit character of the gradient regularization (20): it is a full matrix, due to the inverses in Equation (58). This contrasts with the banded nature of matrix $\mathbf{A}_{\text {integral }}$, with a bandwidth controlled by the characteristic length $l_{\mathrm{c}}$.

Replacing Equation (58) into the first block-equation in system (57) yields

$$
\left[\mathbf{K}_{\mathbf{u u}}^{i}+\mathbf{K}_{\mathbf{u} \widetilde{\mathbf{u}}}^{i} \mathbf{A}_{\text {gradient }}\right] \delta \mathbf{u}^{i+1}=-\mathbf{r}_{\text {equil }}^{i} .
$$

From Equation (59), it is clear again that matrix $\mathbf{A}_{\text {gradient }}$ plays in the gradient version of the damage model exactly the same role as matrix $\mathbf{A}_{\text {integral }}$ in the integral version, see Equation (37): it "spreads" the local matrix $\mathbf{K}_{\mathbf{u u}}^{i}$ to account for nonlocality.

\section{SIMPLE UPGRADE OF A LOCAL MODEL}

Nonlocal displacements can be used to upgrade with nonlocal capabilities a "standard" (i.e. for local models) nonlinear FE code in a very simple and straightforward manner. This is especially true for the gradient approach, because the two matrices needed for the regularization - the 
mass matrix $\mathbf{M}$ and the diffusivity matrix $\mathbf{D}$ - are very simple to compute (they are constant, symmetric definite positive matrices) and readily available in any $\mathrm{FE}$ code.

Since matrix $\mathbf{A}_{\text {gradient }}$ in the one-field formulation of Section 4.3 is a full matrix, we think the best option is the two-field formulation of Section 4.2.2. If assembling and/or factorizing the non-symmetric tangent matrix (52) poses difficulties, the linear system (57) can be solved very effectively by means of the block Gauss-Seidel method. To do so, simply rewrite Equation (57) into

$$
\left.\begin{array}{rl}
\mathbf{K}_{\mathbf{u u}}^{i} \delta \mathbf{u}_{k+1}^{i+1} & =-\mathbf{r}_{\text {equil }}^{i}-\mathbf{K}_{\mathbf{u} \widetilde{\mathbf{u}}}^{i} \delta \widetilde{\mathbf{u}}_{k}^{i+1} \\
+c \mathbf{D}) \delta \widetilde{\mathbf{u}}_{k+1}^{i+1} & =\mathbf{M} \delta \mathbf{u}_{k+1}^{i+1}
\end{array}\right\}
$$

where subscripts $k$ and $k+1$ are the counters for the inner Gauss-Seidel iterations. The two lefthand-side matrices in Equation (60) are symmetric positive definite, so a standard Cholesky factorization applies. Once $\mathbf{K}_{\mathbf{u u}}^{i}$ and $\mathbf{M}+c \mathbf{D}$ are factorized (at the beginning of the current equilibrium iteration $i$ and at the beginning of the analysis, respectively), the inner GaussSeidel iterations have a relatively modest computational cost. Linear convergence is expected for these inner iterations $k$. Note, however, that (1) quadratic convergence is obtained for the expensive, outer equilibrium iterations $i$ and (2) the tolerance for the inner $k$ loop is usually not a constant [28]; a large tolerance is allowed for the initial Newton-Raphson iterations and a strict tolerance is only prescribed when the residual $\mathbf{r}_{\text {equil }}^{i}$ tends to zero.

\section{CONCLUDING REMARKS}

Nonlocal displacements can be effectively used to regularize softening damage models. An internal length scale is incorporated into the model, either via the characteristic length $l_{\mathrm{c}}$ of the averaging function (integral-type version) or parameter $c$ in the second-order PDE (gradient version), so pathological mesh dependence is precluded.

In exchange for averaging a vectorial field of 2 or 3 displacement components rather than the usual scalar field (which has a very modest overhead, because the weighting function for the integral version and the mass and diffusivity matrices for the gradient version are constant), the resulting models are mechanically sound and computationally efficient.

For the integral-type regularization, the consistent tangent matrix is much simpler to compute than for the standard approach (nonlocal state variable), because nonlocal interaction between non-adjacent nodes is accounted for by a constant matrix $\mathbf{A}_{\text {integral }}$, and the need for cumbersome double loops in Gauss points is suppressed.

In the gradient approach, the Dirichlet boundary conditions on the regularization partial differential equation have a clear physical interpretation: nonlocal displacements are prescribed to coincide with local displacements in all the boundary. In addition, these simple boundary conditions are closely connected with the insulation condition represented by the usual homogeneous Neumann boundary conditions on the state variable. The choice of finite element shape functions is straightforward, because the two fields (local and nonlocal displacements) are of the same nature. The expression of the consistent tangent matrix is also simpler, thanks to the linear relation between local displacements $\mathbf{u}$ and nonlocal displacements $\widetilde{\mathbf{u}}$. This gradientenhancement of the displacement field is a very simple way to incorporate nonlocality into a finite element code equipped with standard (i.e. local models) nonlinear capabilities. 


\section{REFERENCES}

1. J. Lemaitre and J.-L. Chaboche. Mechanics of solid materials. Cambridge University Press, Cambridge, 1990.

2. G. Pijaudier-Cabot and Z.P. Baz̆ant. Nonlocal damage theory. J. Eng. Mech.-ASCE, 118(10):1512-1533, 1987.

3. Z.P. Bažant and G. Pijaudier-Cabot. Nonlocal continuum damage, localization instability and convergence. J. Appl. Mech.-Trans. ASME, 55(2):287-293, 1988.

4. J. Mazars and G. Pijaudier-Cabot. Continuum damage theory - application to concrete. J. Eng. Mech.ASCE, 115(2):345-365, 1989.

5. R. de Borst, J. Pamin, R.H.J. Peerlings, and L.J. Sluys. On gradient-enhanced damage and plasticity models for failure in quasi-brittle and frictional materials. Comput. Mech., 17(1-2):130-141, 1995.

6. A. Huerta and G. Pijaudier-Cabot. Discretization influence on regularization by two localization limiters. J. Eng. Mech.-ASCE, 120(6):1198-1218, 1994.

7. Z.P. Bažant and F.B. Lin. Nonlocal smeared cracking model for concrete fracture. J. Eng. Mech.-ASCE, 114(11):2493-2510, 1988.

8. C. Comi. A non-local model with tension and compression damage mechanisms. Eur. J. Mech. A-Solids, 20(1):1-22, 2001.

9. M. Jirásek. Nonlocal models for damage and fracture: comparison of approaches. Int. J. Solids Struct., 35(31-32):4133-4145, 1998.

10. C. Comi. Computational modelling of gradient-enhanced damage in quasi-brittle materials. Mech. Cohesive-Frict. Mater., 4(1), 1999.

11. A. Rodríguez-Ferran, I. Morata, and A. Huerta. Efficient and reliable nonlocal damage models. Comput. Methods Appl. Mech. Eng., 193(30-32):3431-3455, 2004.

12. Z.P. Bažant and M. Jirásek. Nonlocal integral formulations of plasticity and damage: survey of progress. J. Eng. Mech.-ASCE, 128(11):1119-1149, 2002.

13. R. de Borst, L.J. Sluys, H.-B. Mülhaus, and J. Pamin. Fundamental issues in finite element analysis of localization of deformation. Eng. Comput., 10:99-121, 1993.

14. J. Mazars, G. Pijaudier-Cabot, and C. Saouridis. Size effect and continuous damage in cementitious materials. Int. J. Fract., 51(2):159-173, 1991.

15. P. Pegon and A. Anthoine. Numerical strategies for solving continuum damage problems involving softening: application to the homogenization of masonry. In Proceedings of the Second International Conference on Computational Structures Technology, Athens, 1994.

16. A. Rodríguez-Ferran and A. Huerta. Error estimation and adaptivity for nonlocal damage models. Int. J. Solids Struct., 37(48-50):7501-7528, 2000.

17. H. Askes and L.J. Sluys. Explicit and implicit gradient series in damage mechanics. Eur. J. Mech. A-Solids, 21(3):379-390, 2002.

18. R.H.J. Peerlings, M.G.D. Geers, R. de Borst, and W.A.M. Brekelmans. A critical comparison of nonlocal and gradient-enhanced softening continua. Int. J. Solids Struct., 38(44-45):7723-7746, 2001.

19. C. Polizzotto. Gradient elasticity and nonstandard boundary conditions. Int. J. Solids Struct., 40(26):7399-7423, 2003.

20. P. Lancaster and K. Salkauskas. Surfaces generated by moving least squares methods. Math. Comput., 37(155):141-158, 1981.

21. A. Huerta and S. Fernández-Méndez. Locking in the incompressible limit for the Element Free Galerkin method. Int. J. Numer. Methods Eng., 51(11):1361-1383, 2001.

22. A. Simone, H. Askes, R. H. J. Peerlings, and L. J. Sluys. Interpolation requirements for implicit gradientenhanced continuum damage models. Commun. Numer. Methods Eng., 19(7):563-572, 2003.

23. C.Q. Ru and E.C. Aifantis. A simple approach to solve boundary-value problems in gradient elasticity. Acta Mech., 101(1-4):59-68, 1993.

24. T. Belytschko, W.K. Liu, and B. Moran. Nonlinear finite elements for continua and structures. John Wiley \& Sons, Chichester, 2000.

25. G. Pijaudier-Cabot and A. Huerta. Finite element analysis of bifurcation in nonlocal strain softening solids. Comput. Methods Appl. Mech. Eng., 90(1-3):905-919, 1991

26. M. Jirásek and B. Patzák. Consistent tangent stiffness for nonlocal damage models. Comput. Struct., 80(14-15):1279-1293, 2002.

27. A. Rodríguez-Ferran and A. Huerta. Adapting Broyden method to handle linear constraints imposed via Lagrange multipliers. Int. J. Numer. Methods Eng., 46(12):2011-2026, 1999.

28. C.T. Kelley. Iterative methods for linear and nonlinear equations, volume 16 of Frontiers in Applied Mathematics. Society for Industrial and Applied Mathematics, Philadelphia, 1995. 